\section{Better by half}

\section{Nature Photon. 2, 105-109 (2008)}

Conventional photovoltaics produce electricity by generating electron-hole pairs in a semiconductor through the absorption of light. For light that contains a broad spectrum of wavelengths, such as sunlight, the efficiency of this process is limited by the fact that each photon absorbed will usually only generate an amount of electrical energy equivalent to the semiconductor's bandgap, even if the energy of the photon is much greater than the bandgap. But Dolf Timmerman and colleagues suggest this efficiency could be improved - by 'cutting' these photons in two.

The authors demonstrate the principle of the approach by measuring the photoluminescent efficiency of a matrix of silicon nanocrystals and erbium ions embedded in silica. Their results suggest that when a nanocrystal absorbs a photon whose energy is more than twice its bandgap, it can transfer around half of this absorbed energy to a second nearby crystal or erbium ion, by a process known as spaceseparated quantum cutting.

\section{Warp drive}

\section{Phys. Rev. D77, 015003 (2008)}

In the final few months before the turn-on of the Large Hadron Collider at CERN, theorists are busy refining their predictions for what might be seen in the machine's high-energy protonproton collisions. Kaustubh Agashe and colleagues have focused on the signature of the 'Kaluza-Klein gluon' - a particle whose discovery could signal the existence of a warped extra dimension of space, as proposed by Lisa Randall and Raman Sundrum.

Finding the KK gluon won't be easy: it's 'proton-phobic', so difficult to produce, and, although it couples strongly to the top quark, its decay to a top-antitop pair is broad and hard to distinguish from those top pairs generated in more mundane, standard-model processes. The trick, say Agashe et al., will be to find a so-called forward-backward asymmetry, based on the polarization of the top quarks.

This asymmetry arises because the KK gluon would produce preferentially top quarks with right-handed polarization. In contrast, the quantum-chromodynamics mechanism of the standard model produces equal numbers of left-handed and right-handed pairs.

A KK gluon with a mass of up to $4 \mathrm{TeV}$ could be revealed in about 100 inversefemtobarns of data, which will probably be accumulated in the first few years of running the LHC.

\title{
Forty plus
}

\section{J. Magn. Reson.}

doi:10.1016/j.jmr.2007.12.008 (2007)

Narrow resonance lines with a healthy

signal-to-noise ratio are desirable features of NMR spectra. In typical applications, this means that the magnetic field in which the sample is immersed should be strong and homogeneous. In the early days of NMR, these fields were commonly produced by electromagnets, until, 40 years ago, superconducting magnets started to take over. State-of-the-art magnets produce magnetic flux densities of close to $22 \mathrm{~T}$, but current technology is now close to its limits.

The route to even higher fields requires a new approach - or an old one, as Zhehong Gan and colleagues demonstrate. They have performed NMR experiments using electromagnets and hybrid magnets - which combine superconducting and resistive magnets - in fields of up to $40 \mathrm{~T}$. Experiments in even higher fields have been reported before, but were limited

\section{Heated debate}

\section{Icarus 193, 213-223 (2008)}

In 2005 the Cassini spacecraft flew past Enceladus, the sixth largest of Saturn's many moons. In the remarkable images beamed back to Earth was evidence of a water-rich plume ejected from the moon's surface and of significant heat emanating from the south-polar region. Radiogenic heating can account for some, but not all, of that liberated energy; tidal heating is in the frame as the likely source of the rest.

In earlier work, Jennifer Meyer and Jack Wisdom showed that if Enceladus is in fact in tidal equilibrium, then the heating by this method is not sufficient to explain the observations either. Now they consider whether some past instability, such as a period of chaotic variation in the moon's eccentricity - leading to an increased rate of heating - could be the explanation.

Enceladus' history is complicated, and the authors have simulated the dynamics of its orbit taking into account orbital resonances with neighbouring by the poor stability of powered magnets and, consequently, broad resonance lines. But Gan et al. have put together a suite of techniques for compensating field inhomogeneities to reach spectral resolutions approaching those achieved in conventional superconducting magnets.

\section{Phase force}

\section{Phys. Rev. Lett. 100, 013602 (2008)}

It is well known that light carries momentum. This is exploited in so-called optical tweezers, which use optical gradients to push small particles from regions of high to low light intensity. Less well known, however, is that spatial variations in an optical field's phase can also generate a force on a particle - as Yohai Roichman and colleagues show.

Using shape-phase holography, the authors generate a variety of optical fields with differently shaped phase gradients. They show that, for a linear phase gradient, a water-suspended glass microsphere is propelled along the gradient, similar to its behaviour in an intensity gradient.

More significantly, by combining the effects of intensity and phase, more complex control can be exerted, demonstrated by the circular motion of a microsphere in a ring-like intensity trap with an azimuthal phase gradient.

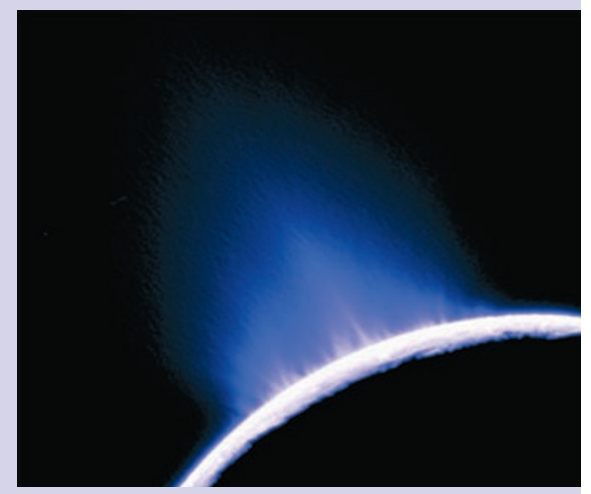

saturnian satellites Mimas and Dione. Curiously, although their calculations suggest the possibility of chaotic variations in the future, there is no sign of such behaviour in the past. It also seems that Enceladus is indeed close to its equilibrium eccentricity.

The mystery remains - perhaps, say the authors, some form of nonequilibrium thermal behaviour is behind it. 